\title{
Artificial Inoculation of Wheat for Selecting Resistance to Stagonospora Nodorum Blotch
}

\author{
Christina Cowger, USDA-ARS, Department of Plant Pathology, North Carolina State University, Raleigh 27695;
} and J. Paul Murphy, Crop Science Department, North Carolina State University, Raleigh 27695

\begin{abstract}
Cowger, C., and Murphy, J. P. 2007. Artificial inoculation of wheat for selecting resistance to Stagonospora nodorum blotch. Plant Dis. 91:539-545.

In the eastern United States, natural epidemics of Stagonospora nodorum blotch (SNB) are not consistently severe enough to facilitate substantial progress in breeding moderately resistant cultivars of soft red winter wheat. We compared three artificial inoculation methods to natural inoculum in a field experiment involving seven wheat (Triticum aestivum) cultivars with varying levels of SNB resistance. Artificial inoculation methods were: Phaeosphaeria nodorum conidia applied by atomization to three- to four-leaf wheat in early winter, $P$. nodorum conidia applied by atomization at boot stage in late spring, and $P$. nodorum-infected wheat straw applied in early winter. The experiment was conducted at Kinston and Plymouth, NC, in 2003-2004, 2004-2005, and 2005-2006, and all treatments had three replicates. Percent diseased canopy was assessed and comparisons were made using disease severity at a single date (early to soft dough stage) and area under the disease progress curve (AUDPC). The relative resistance level of cultivars was consistent across sites, years, and inoculum methods, although the rankings of moderately susceptible and susceptible cultivars were sometimes switched. On average, late spores and straw caused significantly more disease than early spores or natural inoculum $(P \leq 0.05)$. Biplot analysis indicated that all artificial methods had a higher mean capacity to discriminate among cultivars than did natural inoculum $(P \leq 0.05)$. On average, artificial inoculation increased the capacity of environments to separate wheat cultivars by SNB resistance.
\end{abstract}

Additional keywords: cereals, principal component analysis

Phaeosphaeria nodorum (E. Muller) Hedjaroude (anamorph = Stagonospora nodorum (Berk.) Castellani \& E.G. Germano) causes Stagonospora nodorum blotch (SNB), a disease of wheat (Triticum aestivum) leaves, stems, and glumes. SNB occurs persistently, but with varying degrees of severity, in the eastern U.S. soft red winter wheat region. Acceptable levels of partial resistance are available in adapted soft red winter wheat lines, yet many widely grown varieties are susceptible (4). Progress in breeding for SNB resistance in this region has been modest (23) due to a lack of continuous selection pressure, low heritability of resistance, and sporadic natural epidemics $(9,10)$.

$P$. nodorum has both sexual and asexual stages, and populations from diverse geographic regions have high levels of gene and genotypic diversity $(11,12)$ and a balance of mating types $(6,24)$ consistent with random mating. Uncertainty remains about

Corresponding author: C. Cowger

E-mail: christina_cowger@ncsu.edu

Accepted for publication 10 November 2006.

doi:10.1094/PDIS-91-5-0539

This article is in the public domain and not copyrightable. It may be freely reprinted with customary crediting of the source. The American Phytopathological Society, 2007. the relative importance of seed infections, pycnidiospores, and ascospores from previous crop debris in establishing SNB epidemics $(6,11,12,17,22)$. The pathogen may be seedborne in a high percentage of wheat seed samples, and the percentage of seed that is infected can vary considerably $(3,7,17,21,27)$.

An effective and practical artificial field inoculation technique would facilitate breeding for resistance to $P$. nodorum $(8,10)$, but little is known about the best timing for such inoculations. Williams and Jones (27) found that severity of seed infection by $P$. nodorum was greatest when inoculations were performed at $1 / 2$ - to $3 / 4-$ head emergence, and declined steadily when plants were inoculated at later growth stages. Wainshilbaum and Lipps (25) found that the growth stage of winter wheat significantly affected its susceptibility to $P$. nodorum, with leaf blotch severity increasing with increasing plant age at time of inoculation. This effect was observed at a range of temperatures $(19,24$, and $29^{\circ} \mathrm{C}$ ) in controlled environment chambers.

Researchers have considered the number of isolates advisable for artificial inoculation of breeding materials $(2,15,16)$. In general, $P$. nodorum interacts quantitatively with wheat $(8,13)$, and major genes for virulence or resistance have not been reported in this pathosystem. Significant cultivar-by-isolate interactions have been found $(1,2,14,19,20)$, although the magnitude of the interaction was generally small relative to the main effects, and nonsignificant interactions have also been observed (16). Fraser et al. (10) observed a masking of host genetic variation after inoculation with a single isolate in one site-year. Concluding that field inoculations with selected $P$. nodorum isolates at boot stage did not distinguish more and less resistant host cultivars better than natural inoculum, they suggested investigation of naturally infected wheat straw as an inoculum source.

Currently, soft red winter wheat in some breeding nurseries is inoculated at boot stage (Zadoks GS 40-49) with P. nodorum conidial suspensions. However, many breeding programs rely solely on natural epidemics. The objective of this study was to assess the efficacy of various artificial inoculation methods. Three methods of artificial inoculation ( $P$. nodorum-infected wheat straw applied in the fall, $P$. nodorum conidia applied in the fall, and $P$. nodorum conidia applied in the spring) were compared with each other and with natural inoculum. The criteria for evaluation of an artificial inoculation method were its capacity to discriminate among cultivars, its accuracy relative to natural inoculum in ranking cultivars, its representativity of the average inoculation environment in the experiment, and its practicability for breeding programs. The study also allowed us to examine the influence of disease severity on yield and test weight.

\section{MATERIALS AND METHODS}

Establishment of field plots. Field plots were planted on 21 October 2003, 22 October 2004, and 20 October 2005 at the Cunningham Research and Extension Center in Kinston, NC, and on 11 November 2003, 9 November 2004, and 3 November 2005 at the Tidewater Research Station in Plymouth, NC. The 2003-2004, 2004 2005, and 2005-2006 field seasons will hereafter be referred to as 2004,2005 , and 2006, respectively. All trials were planted in conventionally tilled fields following corn or soybeans, with no wheat debris in evidence. Interspersed in a checkerboard pattern among the wheat plots were noninoculated buffer plots of barley, which minimized interplot interference. Borders of barley were planted around the experiment. No symptoms of SNB infection were observed in the barley buffer plots. 
After end-trimming, plots measured $3.1 \mathrm{~m}$ in length, and their width was $1.7,1.2$, and $1.5 \mathrm{~m}$ in the 3 years, respectively. Standard commercial practices appropriate to each site were followed with respect to soil preparation, fertilization, and applications of herbicides and insecticides (26).

The experiment had a randomized complete block design, with five (2004) or seven (2005 and 2006) host cultivars, each subjected to each of four inoculation methods (natural inoculum, early spores, late spores, and infected straw). All hostinoculation method combinations were replicated three times. Host cultivars (Table 1) were chosen from among commercially planted varieties adapted to North Carolina and represented a spectrum of levels of SNB resistance (4,5). In 2005 and 2006, cultivars AGS 2000 and USG 3650 were added to the experiment to include higher levels of susceptibility (5).

Before planting, seed of all cultivars was treated with difenoconazole + metalaxyl (Dividend Xtreme; Syngenta Crop Protection, Greensboro, NC) at a rate of $1.3 \mathrm{ml}$ product per $\mathrm{kg}$ seed, with the fungicide diluted 1:8 with tap water. The purpose of the seed treatment was to neutralize possible differences among seed lots in degree of $P$. nodorum infection.

Inoculum preparation. Straw inoculum. Wheat straw infected with $P$. nodorum was collected in August 2003, June 2004, and June 2005 from fields at the Cunningham Research and Extension Center in which symptoms of SNB had been observed. In 2004, the straw was fed through a chopper, producing pieces of 5 to $10 \mathrm{~cm}$ in length. In 2005 and 2006, the straw was baled but not chopped. Each year, 500-g portions of straw were prepared for each plot to be inoculated.

Spore inoculum. All $P$. nodorum isolates used to produce spore inoculum were derived from wheat leaves collected in the field and incubated in petri dishes over moist filter paper. The resulting monopycnidial isolates were transferred as cirrhi and incubated on potato dextrose agar (PDA). In 2004, nine isolates were used for inoculation. The isolates originated from three host cultivars; seven isolates came from Kinston and two from Plymouth. In 2005, 10 isolates were used in the early spore inoculations, but only two and three isolates produced inoculum for the late spore inoculations at Kinston and Plymouth, respectively, due to lack of sporulation by other isolates. In 2006, seven and nine isolates were used in the early inoculations in Kinston and Plymouth, respectively, and five isolates for the late inoculations at both sites.

For inoculum preparation, isolates were grown on V8 agar plates (200 ml V8 juice, $3 \mathrm{~g} \mathrm{CaCO}_{3}, 15 \mathrm{~g}$ agar per liter of medium) under black light at 22 to $24^{\circ} \mathrm{C}$ for 12 days. Plates were flooded with sterile $\mathrm{dH}_{2} \mathrm{O}$ and rubbed with a bent glass rod. Spore suspensions were decanted and concentrations were measured with a hemacytometer. In 2004, 375 to $400 \mathrm{ml}$ were applied per plot, with a concentration of $10^{6}$ spores per ml. In 2005 and 2006, each plot received 240 to $300 \mathrm{ml}$ of spore suspension, depending on inoculum availability, with concentrations of $5.3 \times 10^{7}$ to $1.5 \times$ $10^{8}$ spores per $\mathrm{ml}$. Over the 2 years, this resulted in a 2.8-fold difference in the lowest versus the highest number of spores applied to a plot.

Inoculation. Applications of straw and early spores were made when varieties were at the three- to four-leaf stage (Zadoks GS 13-14). In Kinston, this occurred on 18 November 2003, 18 November 2004, and 21 November 2005, and in Plymouth on 9 December 2003, 7 December 2004, and 13 December 2005. Late spore inoculations were performed at boot stage (Zadoks GS 41-49) in Kinston on 5 April 2004, 14 April 2005, and 6 April 2006, and in Plymouth on 12 April 2004,
18 April 2005, and 19 April 2006. In 2004 and for the early inoculation in 2005, spores were applied evenly to all plants in each plot with a hand-operated spray bottle. Starting with the late inoculation in 2005, spores were applied evenly to all plots with a backpack sprayer (RootLowell Mfg., Lowell, MI) at 65 psi. Straw inoculation consisted of spreading $500 \mathrm{~g}$ of straw evenly throughout a plot by hand.

After early spore applications in 2004 and 2005, plastic tarps were placed over plots and weighted at the edges to enhance incubation. In 2004, tarps remained on plots for 3 days after early spore applications at both sites, but in 2005 tarps were removed after 1 day at Kinston or 2 days at Plymouth due to warm temperatures. After late spore applications, plots were tarped in the same manner in 2004 for 1 and 3 days at Kinston and Plymouth, respectively, but were not tarped in 2005 after the late spore application at either site, again due to warm temperatures. No tarping was conducted in 2006.

Disease assessment and harvest. Disease was assessed in each experiment on three dates, except for Kinston in 2005, where only one assessment was made, and Plymouth in 2006, where only two assessments were made due to late disease development. The assessment dates at Kinston were 30 April, 7 May, and 17 May 2004; 13 May 2005; and 3 May, 10 May, and 17 May 2006. The dates at Plymouth were 3 May, 13 May, and 20 May 2004; 19 May, 26 May, and 1 June 2005; and 18 May and 24 May 2006. Crop maturity ranged between Zadoks GS 77 (late milk) and 87 (hard dough) during SNB assessment. Assessments, which were all conducted by the same observer, were in the form of percent SNB-diseased leaf area on a whole-plot basis, and did not take glume symptoms into account.

All plots were harvested by combine each year, and moisture content and test

Table 1. Percent severity of Stagonospora nodorum blotch ${ }^{\mathrm{w}}$ averaged across site-years following natural and artificial inoculation of soft red winter wheat varieties in North Carolina in 2004 to $2006^{\mathrm{x}}$

\begin{tabular}{|c|c|c|c|c|c|c|c|}
\hline \multirow[b]{2}{*}{ Cultivar } & \multirow{2}{*}{$\begin{array}{l}\text { P.I. / PVP / } \\
\text { tagging nbr. }\end{array}$} & \multirow{2}{*}{$\begin{array}{c}\text { Resistance } \\
\text { level }^{\mathbf{z}}\end{array}$} & \multicolumn{5}{|c|}{ Inoculation method ${ }^{y}$} \\
\hline & & & Natural & Early spores & Late spores & Straw & Mean \\
\hline NC Neuse & 200400303 & MR & $16.6 \mathrm{a}$ & $20.0 \mathrm{a}$ & $28.8 \mathrm{a}$ & $27.1 \mathrm{a}$ & $23.1 \mathrm{a}$ \\
\hline Vigoro Tribute & 632689 & MR & $18.9 \mathrm{a}$ & $23.4 \mathrm{a}$ & $33.1 \mathrm{a}$ & $30.9 \mathrm{a}$ & $26.6 \mathrm{a}$ \\
\hline $\mathrm{P} 26 \mathrm{R} 12$ & 200200234 & MR & $26.9 \mathrm{~b}$ & $32.2 \mathrm{~b}$ & $41.1 \mathrm{~b}$ & $40.4 \mathrm{~b}$ & $35.1 \mathrm{~b}$ \\
\hline USG 3209 & 200100127 & MS & $29.7 \mathrm{~b}$ & $34.2 \mathrm{bc}$ & $45.2 \mathrm{bc}$ & $42.6 \mathrm{bc}$ & $37.9 \mathrm{bc}$ \\
\hline USG 3650 & W711 & $\mathrm{S}$ & $30.0 \mathrm{~b}$ & $33.4 \mathrm{bc}$ & $44.8 \mathrm{bc}$ & $46.0 \mathrm{bcd}$ & $38.5 \mathrm{bc}$ \\
\hline P26R24 & 200000275 & MS & $32.8 \mathrm{~b}$ & $39.9 \mathrm{c}$ & $48.4 \mathrm{c}$ & $48.2 \mathrm{~cd}$ & $42.3 \mathrm{c}$ \\
\hline AGS 2000 & 612956 & $\mathrm{~S}$ & $32.4 \mathrm{~b}$ & $40.3 \mathrm{c}$ & $49.8 \mathrm{c}$ & $51.1 \mathrm{~d}$ & $43.4 \mathrm{c}$ \\
\hline \multirow[t]{2}{*}{ Mean } & & & 26.7 & 31.9 & 41.6 & 40.9 & 35.3 \\
\hline & & & A & A & B & B & \\
\hline
\end{tabular}

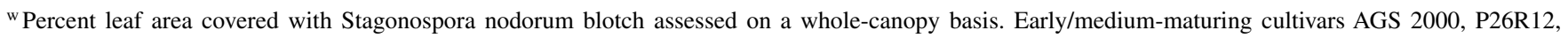
P26R24, and USG3209 were at soft dough stage, and late-maturing cultivars NC Neuse, Tribute, and USG 3650 were at late milk/early dough stage. Assessment dates were 7 May 2004, 13 May 2005, and 10 May 2006 at Kinston, and 13 May 2004, 26 May 2005, and 18 May 2006 at Plymouth. All treatments were replicated three times.

${ }^{x}$ Within a column, means followed by the same letter are not different at $P \leq 0.05$. Within the "mean" row, means over the same letter are not different at $P \leq$ 0.05 .

y Natural inoculum or artificial inoculation with spores ("early spores") or straw at the three- to four-leaf stage (Zadoks GS 13-14), or with spores at the boot stage (Zadoks GS 41-49, "late spores"). All treatments were replicated three times.

${ }^{\mathrm{z}}$ From $(4,5)$. 
weight were determined on a grain analysis computer (GAC 2100, Dickey-John Corp., Auburn, IL). Yields were determined by adjusting the grain weight of samples, as measured on a balance, by their moisture content, using a mean moisture content appropriate to the site-year (12.5 to $14 \%)$.

Data analysis. Effects of factors on SNB severity were analyzed using PROC MIXED (SAS Institute, Cary, NC). Cultivar, inoculation method, and their interaction were treated as fixed variables. Environment (site-year) and its interactions with the fixed variables were treated as random variables, as was replicate. The independent variables were AUDPC or single-date disease severity (DS) at a stage of crop maturity common to all four siteyears: soft dough (Zadoks GS 85) for early- and medium-maturing varieties, and early dough (Zadoks GS 83) for latematuring varieties. DS was the severity reading taken on the second date where three assessment dates are listed above for a given site-year, on 13 May 2005 at Kinston, and on 18 May 2006 at Plymouth. DS was used in addition to AUDPC in analyzing data because it was available for all environments and because breeding programs are more likely to work with single-date assessments.

Contrasts were requested in PROC MIXED to determine whether artificial inoculation led to greater differences in disease severity between resistance categories than did natural inoculum. The contrasted categories were moderately resistant (MR), 'NC Neuse' and 'Tribute'; moderately susceptible (MS), 'P26R12,' 'USG 3209,' and 'USG 3650'; and susceptible (S), 'P26R24' and 'AGS 2000.' Means were separated using Fisher's protected least significant difference (LSD) test. Data were also analyzed separately by year and site.

The effects of DS and AUDPC on yield and on test weight were analyzed using PROC MIXED, with replicate as a random effect. In addition, the correlation of yield or test weight with DS or AUDPC was assessed with PROC CORR, and yield and test weight were regressed separately on DS or AUDPC across years and separately by year, using SAS PROC REG.

Spearman coefficients of correlation were calculated using SAS PROC CORR. In the correlation analysis, the ranking of cultivar means by inoculation methods within and across site-years was compared with the mean ranking by natural inoculum across site and year. The ranking of each naturally inoculated environment was compared with the ranking of the mean of other naturally inoculated environments, excluding itself.

Biplot analysis was conducted with the GGEBiplot program (28), version 3.7.31, to evaluate inoculation techniques for their capacity to discriminate among cultivars and their representativity of the average environment in the experiment. Representativity of the average environment indicates the degree to which the cultivar rankings produced by a given combination of site, year, and inoculation method reflected mean rankings by all site-year-inoculation method combinations. A biplot is a scatter plot that graphically displays a rank-two matrix that approximates a two-way table, using the first two principal components, $\mathrm{PC} 1$ and PC2. In this case, the rows of the table were cultivars and the columns were environments, with each environment being one of the possible combinations of inoculation treatment, site, and year. Biplots were generated based on an environment-centered model (29)

$$
p_{i j}=y_{i j}-\mu-\beta_{j}=\alpha_{i}+\phi_{i j}
$$

where $p_{i j}$ is an element of a rank-two matrix, $y_{i j}$ is the value of cultivar $i$ in environment $j, \mu$ is the grand mean, $\beta_{j}$ is the environment main effect, $\alpha_{i}$ is the cultivar main effect, and $\emptyset_{i j}$ is the cultivar-byenvironment interaction. The form of singular value decomposition (SVD) used was environment-metric or column-metric preserving (29). This form of SVD resulted in vector lengths for environments that approximated the standard errors of disease severity readings within the environments.

The mean discrimination, or ability to distinguish cultivar resistance levels, of each artificially inoculated environment was compared with that of natural inoculum by averaging vector lengths across site-years. The discriminating ability of an environment corresponds to the length of the vector that extends from the biplot origin to the environment marker (30). A longer vector, indicating a more discriminating environment, approximates a higher standard error among values of a measured trait in a given environment. The representativity of inoculation methods of the average environment in the experiment corresponds to their angular separation from the average environment axis, which passes through the biplot origin and the average or "ideal" environment (30). Mean vector lengths $(\mathrm{mm})$ and angular distances (degrees) of the different inoculation methods were separated using Fisher's protected least significant difference test. Vector lengths in a biplot are intrinsically unitless, but all vectors were taken from the same biplot, and thus were comparable to each other.

\section{RESULTS}

Environments. The years 2004 and 2005 were relatively favorable for wheat production in North Carolina, with statewide mean yields significantly above the 10-year statewide mean (USDA-NASS). In 2004 , there was a moderately severe powdery mildew epidemic (caused by Blumeria graminis f. sp. tritici) in Kinston, although it had no evident impact on stand health. That year in Plymouth, however, severe epidemics of Barley yellow dwarf virus (BYDV), Wheat soilborne mosaic virus (WSBMV), and Wheat spindle streak mosaic virus (WSSMV) caused some uneven stands and stunting, with 'USG 3209' particularly affected. In 2005, conditions for wheat growth in both sites were excellent, as a cool spring led to high mean yields and test weights, and other diseases played minor roles. In 2006, by contrast, wheat stands in both sites were thin, mainly due to Hessian fly attack and periods of waterlogging and drought.

There were significant differences among site-years in SNB severity (data not shown). Mean severity was greatest in 2004 (47\%), intermediate in 2005 (36\%), and least in 2006 (23\%). On average, severity was greater in Plymouth (37\%) than in Kinston (34\%). Fixed and random factors accounted for total experimental variation as follows: cultivar, $17 \%$; environment (site-year), $43 \%$; inoculation method, $13 \%$; cultivar-by-environment interaction, 5\%; cultivar-by-inoculation method interaction, $0 \%$; environment-by-inoculation method interaction, $11 \%$; and replication, $2 \%$.

Effects of cultivar. Cultivars varied significantly for mean disease severity (Table 1). 'NC Neuse' and 'Vigoro Tribute' were the most resistant cultivars within and across inoculation methods. In Figure 1, cultivars toward the right are more susceptible, and those toward the left are more resistant. Of the seven cultivars tested in 2005 and 2006, 'AGS 2000' (G1) and 'P26R24' (G4) were the most susceptible and 'NC Neuse' (G2) the most resistant (Fig. 1).

The effect of cultivar on disease severity and AUDPC did not vary by inoculation method (Table 2). In other words, cultivars performed consistently across inoculation methods with regard to relative degree of resistance. This was also true when data were analyzed separately by site and year except in the case of Plymouth in 2006, where the cultivar-by-inoculation method interaction was significant $(P<0.0001)$ because different inoculation methods switched the rankings of moderately susceptible and very susceptible cultivars with respect to one another (data not shown).

Performance of inoculation methods. Averaged across cultivars and site-years, late spores and straw caused more disease than early spores or natural inoculum (Table 1). The above-mentioned $11 \%$ of total variation accounted for by the interaction of inoculation method and environment was mainly due to the fact that late spores and straw switched ranks among site-years (data not shown).

In Figure 1, "environments" are combinations of inoculation method, year, and site. The interaction of inoculation method with environment can be seen in the clustering of environments by site and year. In 
addition, the relative positions of cultivars and environments in Figure 1 illustrate genotype-by-environment interaction. For example, all the Plymouth environments are on the same side of the horizontal axis as 'P26R24,' while most of the Kinston environments are on the opposite side of the horizontal axis, closer to 'AGS 2000'. This indicates that, on average, Plymouth environments ranked 'P26R24' as more susceptible than 'AGS 2000,' while the reverse was true in Kinston.

Discrimination. For the seven cultivars common to the 16 environments in 2005 and 2006, artificial inoculation was significantly more discriminating than natural inoculation (Fig. 1, Table 3). As Table 1 and the contrast results in Table 2 indicate, the advantage of artificial inoculation over natural inoculum is that there is greater separation between the most and least resistant cultivars (MR versus $S$ ).

When AUDPC was used in the biplot analysis instead of DS, there were no significant differences in discrimination among inoculation methods either for five cultivars common to all 20 environments, or for seven cultivars in 12 environments (data not shown).

Accuracy. The accuracy of an artificial inoculation treatment was defined as the correlation of its ranking with that produced by the mean of naturally inoculated plots. Averaged across site-years, rankings of all artificial methods were significantly and positively correlated with the mean of natural inoculum (Table 4). Using DS, within site-years, most individual treatments were also significantly correlated with the mean of natural inoculum (Table 4). The exceptions were all four treatments in 2004 at Plymouth, where virus diseases were severe. Using AUDPC, several environments were not significantly correlated with the mean of natural inoculum at $P \leq$ 0.05 .

From a practical perspective, an inoculation method should facilitate assignment of cultivars to categories of SNB resistance. The categories of high, medium, and low are likely to be convenient, and can also be designated as moderately resistant (MR), moderately susceptible (MS), and susceptible (S). From Figure 1 and Table 1, those categories can be delineated as follows: $\mathrm{MR}=$ 'NC Neuse' and 'Tribute'; MS = 'P26R12,' 'USG 3209,' and 'USG 3650'; and $S=$ 'P26R24' and 'AGS 2000.' From rankings by DS alone, without respect to whether differences among means were significant, we tallied the category misassignments produced by each inoculation method. For the four site-years with seven cultivars, all inoculation methods always assigned 'NC Neuse' to MR, and 'Tribute' was correctly assigned to MR in all but one case (Kinston 2005, early spores, assigned to MS). The next most resistant cultivar, 'P26R12,' was also misassigned once (to MR by natural inoculum, Kinston 2006). The other cultivars were misassigned in three to five cases each, switching MS for S or vice versa. Overall, natural inoculum, early spores, and late spores produced three category misassignments

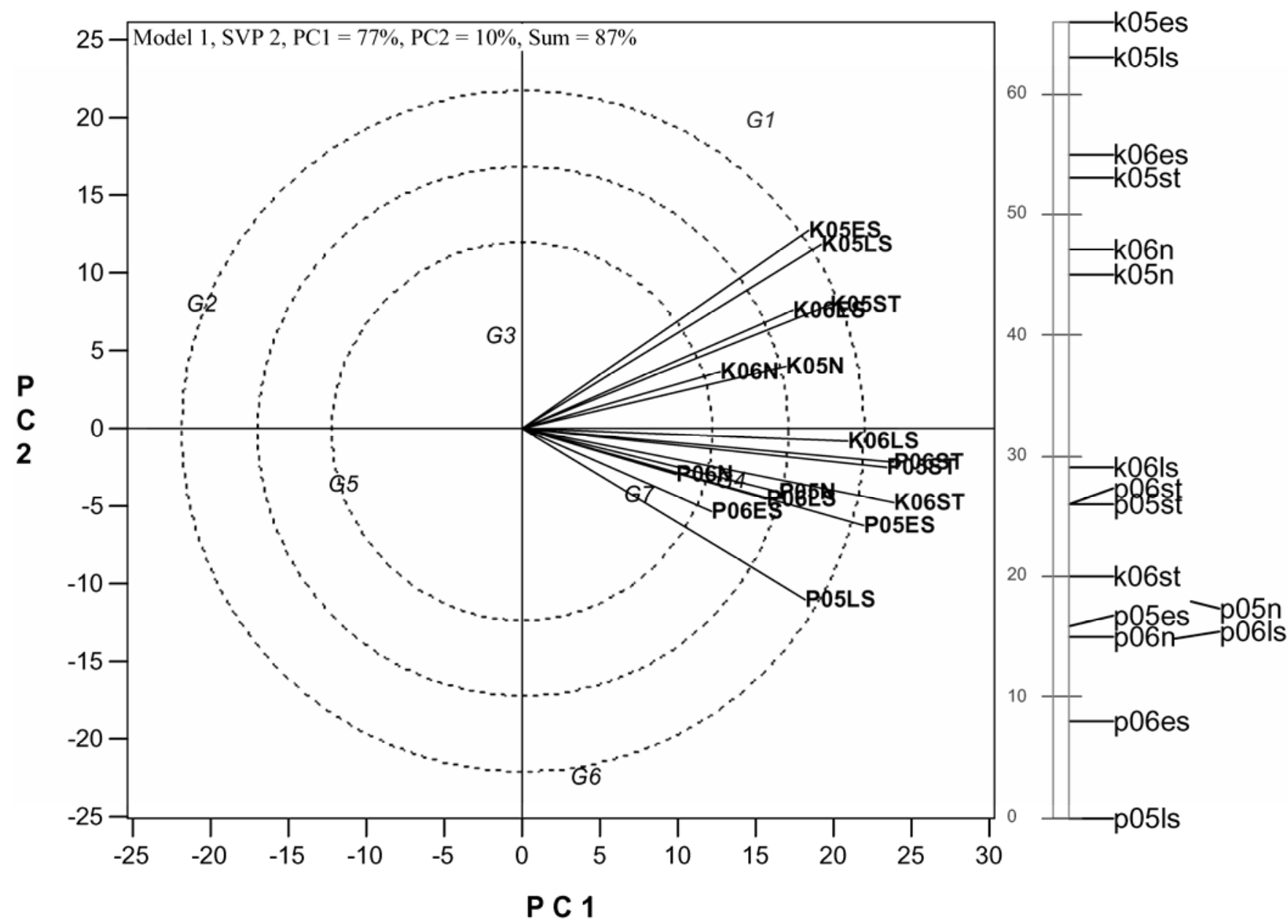

Fig. 1. Biplot depiction of ranking of seven soft red winter wheat cultivars in susceptibility to Stagonospora nodorum blotch, using disease severity at soft dough stage for early/medium-maturing cultivars (G1 = 'AGS 2000,' G3 = 'P26R12,' G4 = 'P26R24,' and G6 = 'USG 3209') and at late milk/early dough stage for later-maturing cultivars (G2 = 'NC Neuse,' G5 = 'Vigoro Tribute,' and G7 = 'USG 3650'). Cultivars toward the right (positive values of PC1) are more susceptible; those toward the left (negative values of PC1) are more resistant. Disease assessments are means of three replicates. Each environment label includes site, year, and inoculation method. Sites: K = Kinston, P = Plymouth. Years: 2004, 2005, and 2006. Inoculation methods: N = natural, ES = early spores (three- to four-leaf stage), LS = late spores (boot stage), $\mathrm{ST}=$ straw (at three- to four-leaf stage). Discrimination of environments corresponds to the length of their vectors from the biplot origin. In the linear map on the right, which is scaled in degrees, environments closer to the horizontal axis $\left(32^{\circ}\right)$ are more representative of the average environment in the experiment. 
each. Straw produced one misassignment (an MS-S switch).

Using AUDPC instead of DS, the only misassignments were switches of cultivars between MS and S categories. There were two for natural inoculum, two for early spores, three for late spores, and two for straw.

Representativity. We analyzed how representative each inoculation method was of the average environment in the experiment, which seemed a reasonable comparison as none of the inoculation methods was especially or consistently uncorrelated with natural inoculum (Table 4). In a biplot, the angular separation of an environment from the horizontal average environment axis (AEA) (29) indicates how well the rankings of that environment reflect the average environment in the experiment. A greater projection away from the AEA, regardless of direction, means that the environment is less representative of the average environment. The horizontal axis in Figure 1 coincides exactly with the AEA. Overall, environments were relatively tightly clustered around the average environment, with a total angular separation of less than 70 degrees among all environments (Fig. 1).

Representativity (R) of artificial inoculation methods is quantified in Table 3 as the mean angular distance of each method from the AEA divided by the mean of naturally inoculated environments. For the seven cultivars tested in 2005 and 2006, the differences in mean representativity among inoculation methods were not significant. This was also true when AUDPC was used in the biplot analysis instead of DS, both with the five cultivars common to all 20 environments, and with the seven cultivars in 12 environments (data not shown), although the relative magnitudes of the means varied.
Yield and test weight. Yield was significantly negatively correlated with DS in 2004 at both sites, but not in the other years at either site (data not shown). Test weight was significantly negatively correlated with DS for four of the six site-years. Neither yield nor test weight varied significantly by cultivar or inoculation method (data not shown).

\section{DISCUSSION}

On average, artificial inoculation by any of our methods increased discrimination among cultivars segregating for resistance to SNB. Fraser et al. (10) found no benefit from supplemental inoculation with selected $P$. nodorum isolates. The discrepancy between our conclusion and theirs may be due to the greater diversity of isolates in our experiment, or of host cultivars in theirs. Seedborne inoculum may also have played a stronger role in their experiment, as their seed was not treated with fungicide.

Statistically, no artificial method was consistently superior to the others in our experiment, either in discrimination or in the correlation of its ranking of cultivars to the mean ranking produced by natural inoculum. However, straw more consistently assigned cultivars correctly to the categories of "moderately resistant," "moderately susceptible," and "susceptible" than did other inoculation methods.

Moreover, infected wheat straw has several advantages over spores for artificial inoculation. It provides a genetically diverse pathogen population, which can only be achieved with considerable labor in the case of spores. Straw produces inoculum over an extended period, and is therefore neutral with respect to maturity differences among cultivars, unlike spores applied at boot stage. Also, the total effort involved in baling, storing, and applying straw is substantially less than that re- quired to derive, maintain, and increase isolates, and apply spores. This is especially relevant because SNB resistance is only one among many traits of interest in breeding programs. A disadvantage of straw inoculum is that it contains seed and produces volunteer plants, and thus should not be applied where pure seed is to be harvested.

Discrimination of SNB susceptibility is likely to be enhanced if, during stem elongation, there is precipitation or irrigation of sufficient force to transfer secondary inoculum up the emerging crop canopy. (Applications of spores during boot stage are less dependent on rain-mediated secondary spread.) For example, in the period of March to May 2004, there were only one or two occasions per month at the Plymouth test site with $\geq 1.27 \mathrm{~cm}$ of rain-

Table 3. Mean discrimination (D) ${ }^{\mathrm{x}}$ and representativity $(\mathrm{R})^{\mathrm{y}}$ shown in Figure 1 of methods for natural and artificial inoculation with Phaeosphaeria nodorum at Kinston and Plymouth, NC, in 2005 and $2006^{2}$

\begin{tabular}{lcc}
\hline $\begin{array}{l}\text { Inoculation } \\
\text { method }\end{array}$ & D & R \\
\hline Natural inoculum & $1.00 \mathrm{a}$ & $1.00 \mathrm{a}$ \\
Spores early & $1.34 \mathrm{~b}$ & $1.64 \mathrm{a}$ \\
Spores late & $1.40 \mathrm{~b}$ & $1.41 \mathrm{a}$ \\
Straw & $1.61 \mathrm{~b}$ & $0.76 \mathrm{a}$ \\
\hline
\end{tabular}

${ }^{x}$ Discrimination corresponds to the distance $(\mathrm{mm})$ from the biplot origin in Figure 1 to the treatment markers.

${ }^{y}$ Representativity of the average environment in the experiment corresponds to the angular distance (degrees) of a treatment marker from the horizontal axis in Figure 1; lower values indicate greater representativity.

${ }^{\mathrm{z}}$ Values for inoculation methods are divided by the mean value for natural inoculum and thus are unitless. Within a column, means followed by the same letter are not different $(P \leq 0.05)$ by Fisher's protected least significant difference test.

Table 2. Analysis of variance of effects of cultivar and inoculation method on disease severity following natural or artificial inoculation with Phaeosphaeria nodorum at Kinston and Plymouth, NC, in 2004 to 2006

\begin{tabular}{|c|c|c|c|c|c|c|}
\hline & \multicolumn{3}{|c|}{ Disease severity $^{w}$} & \multicolumn{3}{|c|}{ AUDPC $^{x}$} \\
\hline & df & $F / t$ value & $P>F$ & df & $F / t$ value & $P>F$ \\
\hline \multicolumn{7}{|l|}{ Source of variation } \\
\hline Cultivar & 6 & 16.67 & $<0.0001$ & 6 & 11.12 & $<0.0001$ \\
\hline Inoculation method & 3 & 8.10 & 0.002 & 3 & 6.16 & 0.009 \\
\hline Cultivar*inoculation method & 18 & 0.55 & 0.92 & 18 & 0.63 & 0.86 \\
\hline \multicolumn{7}{|c|}{ Estimates: severity caused by artificial inoculation compared with natural inoculation ${ }^{z}$} \\
\hline S vs. MR & 78 & 2.18 & 0.033 & 60 & 1.98 & 0.052 \\
\hline MS vs. MR & 78 & 0.96 & 0.34 & 60 & 0.89 & 0.38 \\
\hline MS vs. S & 78 & 1.41 & 0.16 & 60 & 1.26 & 0.21 \\
\hline
\end{tabular}

${ }^{\mathrm{w}}$ Assessment of percent leaf area covered with Stagonospora nodorum blotch on a whole-canopy basis. Early/medium-maturing cultivars AGS 2000, P26R12, P26R24, and USG3209 were at soft dough stage, and late-maturing cultivars NC Neuse, Tribute, and USG 3650 were at late milk/early dough stage. Assessment dates were 7 May 2004, 13 May 2005, and 10 May 2006 at Kinston, and 13 May 2004, 26 May 2005, and 18 May 2006 at Plymouth. All treatments were replicated three times.

${ }^{x}$ Based on DS and additional assessments in Kinston on 30 April and 17 May 2004, and 3 May and 17 May 2006; and at Plymouth on 3 May and 20 May 2004; 19 May and 1 June 2005; and 24 May 2006. All treatments were replicated three times.

y Natural inoculum or artificial inoculation with spores ("early spores") or straw at the three- to four-leaf stage (Zadoks GS 13-14), or with spores at the boot stage (Zadoks GS 41-49, "late spores"). All treatments were replicated three times.

${ }^{\mathrm{z}}$ A significant result indicates that the difference in disease severity between cultivars with the indicated levels of resistance was greater following artificial inoculation than following natural inoculation. S = susceptible ('AGS 2000' and 'P26R24'); MS = moderately susceptible ('P26R12,' 'USG 3209,' and 'USG 3650'); and MR = moderately resistant ('NC Neuse' and 'Vigoro Tribute'). 
fall on a single day. At Kinston, $\geq 1.27 \mathrm{~cm}$ of rainfall in a day occurred once in March, four times in April, and five times in May. Average discrimination of test environments at Kinston was significantly higher than at Plymouth in 2004 ( $P=$ 0.0007).

External factors specific to sites, such as other diseases, can confound efforts to rank cultivars by SNB resistance. This explains the moderate amount of clustering of inoculation treatments by site-year in Figure 1. In 2004, our results were confounded in Plymouth by severe epidemics of BYDV and WSSMV, and possibly also by drought. Both viral diseases can cause stunting, which in turn could elevate SNB severity on upper plant parts, as SNB inoculum is rain-splashed. Viral diseases may also increase cereal host susceptibility to SNB in other ways (18; C. Cowger, personal observation). The differential impact of these viral diseases likely explained the overall lack of correlation with mean natural inoculum rankings (Table 4). Of course, confounding factors can be a problem whether epidemics are naturally or artificially induced.

Single-date disease assessments gave generally similar results to AUDPC when evaluating the discrimination and represen- tativity of inoculation techniques, although the difference between the discrimination of natural inoculum and that of artificial inoculation was not significant with AUDPC. This was true even though our cultivars spanned a range of maturities of about 10 days. Most disease resistance screening programs, which include materials with similar maturity differences, will likely rely on one assessment per season in attempting to make gains from selection.

As described above, the quantity of spores applied early or late to plots in 2005 and 2006 varied by a factor of 2.8 , and inoculations utilized different numbers of isolates due to sporulation problems. Treatments with lower numbers of spores (late inoculations at Kinston and Plymouth in 2005) still had mean disease severity significantly higher than natural inoculum when data were analyzed separately by site-year (data not shown). Cultivar-byisolate interactions were not an issue where smaller numbers of isolates were utilized (two and three isolates, respectively, in the late inoculations at Kinston and Plymouth in 2005), as the cultivar rankings produced by those inoculations were significantly correlated with mean cultivar rankings (Table 4).
Starting with the late spore inoculations in 2005, tarping of plots for incubation was discontinued partly due to warm temperatures, and partly because it was impractical for breeding programs. This change probably accounted for some of the environment-by-inoculation method interaction; for example, the early spore inoculations of 2006 were not tarped, and did not cause significantly more severe disease than did natural inoculum.

Our data suggest that SNB may have a stronger negative impact on test weight than on yield. Similarly, Milus and Chalkley (17) found that while yields did not vary significantly among treatments with different SNB severities, test weights sometimes increased when fungicides were applied to seed and/or foliage. In our experiment, the correlation of yield and test weight with SNB severity was inconsistent, indicating that other disease and environmental factors were sometimes more important.

In conclusion, our results support routine artificial inoculation with $P$. nodorum to facilitate selection of cultivars with high levels of partial resistance to SNB. Infected wheat straw is at least as effective as other forms of artificial inoculation, and is likely to be more convenient.

Table 4. Spearman coefficients and $P$ values for correlation of wheat cultivar rankings by three artificial Phaeosphaeria nodorum inoculation methods with mean ranking by natural inoculum at two sites in North Carolina in 2004 to 2006

\begin{tabular}{|c|c|c|c|c|c|c|c|}
\hline \multirow[b]{2}{*}{ Year } & \multirow[b]{2}{*}{ Site } & \multirow[b]{2}{*}{ Inoculum ${ }^{y}$} & \multirow[b]{2}{*}{$\mathbf{n}^{\mathbf{z}}$} & \multicolumn{2}{|c|}{ Disease severity $^{w}$} & \multicolumn{2}{|c|}{ AUDPC $^{x}$} \\
\hline & & & & Corr coeff & $P$ value & Corr coeff & $P$ value \\
\hline \multirow[t]{8}{*}{2004} & Kinston & Natural & 5 & 0.90 & 0.037 & 0.60 & 0.285 \\
\hline & & Early spores & 5 & 0.90 & 0.037 & 0.70 & 0.188 \\
\hline & & Late spores & 5 & 0.90 & 0.037 & 0.70 & 0.188 \\
\hline & & Straw & 5 & 0.90 & 0.037 & 0.70 & 0.188 \\
\hline & Plymouth & Natural & 5 & 0.60 & 0.285 & 0.70 & 0.188 \\
\hline & & Early spores & 5 & 0.60 & 0.285 & 0.80 & 0.104 \\
\hline & & Late spores & 5 & 0.80 & 0.104 & 0.90 & 0.037 \\
\hline & & Straw & 5 & 0.50 & 0.391 & 0.90 & 0.037 \\
\hline \multirow[t]{8}{*}{2005} & Kinston & Natural & 7 & 0.86 & 0.014 & & \\
\hline & & Early spores & 7 & 0.86 & 0.014 & & \\
\hline & & Late spores & 7 & 0.93 & 0.003 & & \\
\hline & & Straw & 7 & 0.96 & 0.001 & & \\
\hline & Plymouth & Natural & 7 & 0.86 & 0.014 & 0.68 & 0.094 \\
\hline & & Early spores & 7 & 0.96 & 0.001 & 0.96 & 0.001 \\
\hline & & Late spores & 7 & 0.75 & 0.052 & 0.68 & 0.094 \\
\hline & & Straw & 7 & 0.75 & 0.052 & 0.75 & 0.052 \\
\hline \multirow[t]{8}{*}{2006} & Kinston & Natural & 7 & 0.94 & 0.002 & 0.68 & 0.094 \\
\hline & & Early spores & 7 & 0.79 & 0.036 & 0.82 & 0.023 \\
\hline & & Late spores & 7 & 0.93 & 0.003 & 0.96 & 0.001 \\
\hline & & Straw & 7 & 0.87 & 0.012 & 0.96 & 0.001 \\
\hline & Plymouth & Natural & 7 & 0.86 & 0.014 & 0.75 & 0.052 \\
\hline & & Early spores & 7 & 0.89 & 0.007 & 0.89 & 0.007 \\
\hline & & Late spores & 7 & 0.68 & 0.094 & 0.75 & 0.052 \\
\hline & & Straw & 7 & 0.96 & 0.001 & 0.86 & 0.014 \\
\hline \multirow[t]{3}{*}{ Mean } & & Early spores & 7 & 1.00 & $<0.001$ & 0.96 & 0.001 \\
\hline & & Late spores & 7 & 0.96 & 0.001 & 0.96 & 0.001 \\
\hline & & Straw & 7 & 0.96 & 0.001 & 1.00 & $<0.001$ \\
\hline
\end{tabular}

${ }^{w}$ Assessment of percent leaf area covered with Stagonospora nodorum blotch on a whole-canopy basis. Early/medium-maturing cultivars P26R12, P26R24, USG3209, and AGS 2000 were at soft dough stage, and late-maturing cultivars NC Neuse, Tribute, and USG 3650 were at late milk/early dough stage. Assessment dates were 7 May 2004, 13 May 2005, and 10 May 2006 at Kinston, and 13 May 2004, 26 May 2005, and 18 May 2006 at Plymouth. Values are means of three replicates.

${ }^{x}$ Based on disease severity and additional assessments in Kinston on 30 April and 17 May 2004, and 3 May and 17 May 2006; and at Plymouth on 3 May and 20 May 2004; 19 May and 1 June 2005; and 24 May 2006. Values are means of three replicates. AUDPC not available for Kinston 2005.

${ }^{y}$ Artificial inoculation methods are compared with mean rankings across all naturally inoculated environments; individual natural environments are compared with the mean of all other naturally inoculated environments.

${ }^{\mathrm{z}}$ Number of cultivars in the rank comparison. 


\section{ACKNOWLEDGMENTS}

We appreciate the excellent technical assistance of P. Langdon, J. Lovett, J. Özkurt, R. Parks, A. Sutton, D. Yigit, and the staffs of Cunningham Research and Extension Center and Tidewater Research Station. We thank C. Brownie, D. Fraser, and B. Shew for helpful statistical advice and reviews.

\section{LITERATURE CITED}

1. Allingham, E. A., and Jackson, L. F. 1981. Variation in pathogenicity, virulence, and aggressiveness of Septoria nodorum in Florida. Phytopathology 71:1080-1085.

2. Arseniuk, E., and Czembor, P. C. 1999. Hostparasite interactions: Stagonospora nodorum. Pages 63-70 in: Septoria and Stagonospora Diseases of Cereals: A Compilation of Global Research. M. van Ginkel, A. McNab, and J. Krupinsky, eds. CIMMYT, Mexico, D.F.

3. Babadoost, M., and Hebert, T. T. 1984. Incidence of Septoria nodorum in wheat seed and its effects on plant growth and yield. Plant Dis. 68:125-129.

4. Bowman, D. T. 2004. Characteristics of North Carolina Wheat Varieties. Crop Science Research Report No. 211. North Carolina State University, Raleigh. Online publication.

5. Bowman, D. T. 2005. Characteristics of North Carolina Wheat Varieties. North Carolina Measured Crop Performance: Small Grains 2005. Crop Science Research Report No. 214. North Carolina State University, Raleigh. Online publication.

6. Cowger, C., and Silva-Rojas, H. V. 2006. Frequency of Phaeosphaeria nodorum, the sexual stage of Stagonospora nodorum, on winter wheat in North Carolina. Phytopathology $96: 860-866$.

7. Cunfer, B. M. 1978. The incidence of Septoria nodorum in wheat seed. Phytopathology 68:832-835.

8. Eyal, Z. 1999. Breeding for resistance to Septoria and Stagonospora diseases in wheat. Pages 332-344 in: Septoria on Cereals: A Study of Pathosystems. J. A. Lucas, P. Bowyer, and H. M. Anderson, eds. CABI Publishing, New York.

9. Eyal, Z., Scharen, A. L., Prescott, J. M., and van Ginkel, M. 1987. The Septoria Diseases of Wheat: Concepts and Methods of Disease
Management. CIMMYT, Mexico, D.F.

10. Fraser, D. E., Murphy, J. P., Leath, S., and Van Sanford, D. A. 2003. Effect of inoculation with selected isolates of Stagonospora nodorum on field evaluations of host resistance in winter wheat. Plant Dis. 87:1213-1220.

11. Keller, S. M., McDermott, J. M., Pettway, R. E., Wolfe, M. S., and McDonald, B. A. 1997. Gene flow and sexual reproduction in the wheat glume blotch pathogen Phaeosphaeria nodorum (anamorph Stagonospora nodorum). Phytopathology 87:353-358.

12. Keller, S. M., Wolfe, M. S., McDermott, J. M., and McDonald, B. A. 1997. High genetic similarity among populations of Phaeosphaeria nodorum across wheat cultivars and regions in Switzerland. Phytopathology 87:1134-1139.

13. King, J. E., Cook, R. J., and Melville, S. C. 1983. A review of Septoria diseases of wheat and barley. Ann. Appl. Biol. 103:345-373.

14. Krupinsky, J. M. 1997. Aggressiveness of Stagonospora nodorum isolates obtained from wheat in the northern Great Plains. Plant Dis. 81:1021-1031.

15. McDonald, B. A., Mundt, C. C., and Zhan, J. 1999. Population genetics of Mycosphaerella graminicola and Phaeosphaeria nodorum. Pages 77-82 in: Septoria and Stagonospora Disease of Cereals: A Compilation of Global Research. M. van Ginkel, A. McNab, and J. Krupinsky, eds. CIMMYT, Mexico, D.F.

16. Mebrate, S. A., and Cooke, B. M. 2001. Response of wheat cultivars to infection by Stagonospora nodorum isolates/mixture on detached and intact seedling leaves. Euphytica 122:263-268.

17. Milus, E. A., and Chalkley, D. B. 1997. Effect of previous crop, seedborne inoculum, and fungicides on development of Stagonospora blotch. Plant Dis. 81:1279-1283.

18. Pelletier, G. J., Comeau, A., and Couture, L. 1974. Interaction entre le virus de la feuille rouge de l'avoine (BYDV), Septoria avenae et Puccinia coronata sur Avena sativa. Phytoprotection 55:9-12.

19. Scharen, A. L., and Eyal, Z. 1983. Analysis of symptoms on spring and winter wheat cultivars inoculated with different isolates of Septoria nodorum. Phytopathology 73:143-147.

20. Scott, P. R., Benedikz, P. W., and Cox, C. W.
1982. A genetic study on the relationship between height, time of ear emergence, and resistance to Septoria nodorum in wheat. Plant Pathol. 31:45-60.

21. Shah, D. A., and Bergstrom, G. C. 1993. Assessment of seedborne Stagonospora nodorum in New York soft white winter wheat. Plant Dis. 77:468-471.

22. Shah, D. A., Bergstrom, G. C., and Ueng, P. P. 1995. Initiation of Septoria nodorum blotch epidemics in winter wheat by seedborne Stagonospora nodorum. Phytopathology 85:452-457.

23. Shaner, G. 1999. Breeding for resistance to Septoria and Stagonospora blotches in winter wheat in the United States. Pages 127-130 in: Septoria and Stagonospora Diseases of Cereals: A Compilation of Global Research. M. van Ginkel, A. McNab, and J. Krupinsky, eds. CIMMYT, Mexico, D.F.

24. Sommerhalder, R. J., McDonald, B. A., and Zhan, J. 2006. The frequencies and spatial distribution of mating types in Stagonospora nodorum are consistent with recurring sexual reproduction. Phytopathology 96:234-239.

25. Wainshilbaum, S. J., and Lipps, P. E. 1991. Effect of temperature and growth stage of wheat on development of leaf and glume blotch caused by Septoria tritici and $S$. nodorum. Plant Dis. 75:993-998.

26. Weisz, R. 2004. Small Grain Production Guide 2004-05. Crop Science Research Report No. AG-580. North Carolina Cooperative Extension Service, NC State University, Raleigh.

27. Williams, J. R., and Jones, D. G. 1973. Epidemiology of Septoria tritici and S. nodorum. Trans. Br. Mycol. Soc. 61:33-39.

28. Yan, W. 2001. GGEbiplot--A Windows application for graphical analysis of multienvironment trial data and other types of two-way data. Agron. J. 93:1111-1118.

29. Yan, W. 2005. Use of biplot analysis in crop breeding. Pages 7-29 in: Proc. Eastern Wheat Workers Southern Small Grain Workers Conf C. S. Swansons, ed. University of Kentucky, Bowling Green.

30. Yan, W., and Kang, M. S. 2003. GGE Biplot Analysis: A Graphical Tool for Breeders, Geneticists, and Agronomists. CRC Press, Boca Raton, FL. 